\section{(9)}

Aasheim, Henrik; Hydro Aluminium AS, Norway

Abboud, Ali; University of Siegen, Germany

Abdelkader, Ahmed; Enppi, Egypt

Abdulrahim, Faeq; Aluminum Bahrain, Bahrain

Abu-Lebdeh, Taher; North Carolina A\&T State University, United States

Acheampong, Daniel; Volta Aluminium Co. Ltd, Ghana

Adam, Hamish; Boreal Laser, Canada

Adamick, Colton J.; SpaceX, United States

Adhikari, Saikat; Aditya Birla Science and Technology, India

Admal, Nikhil; University of California Los Angeles, United States

Agbo, Dela; Volta Aluminium Co. Ltd, Ghana

Ahlfors, Magnus; Quintus Technologies LLC, United States

Ahmed, A.Rasool Ahmed; Aluminium Bahrain, Bahrain

Aidhy, Dilpuneet S.; University of Wyoming, United States

Ajayi, Oluseyi; Covenant University, Nigeria

Akram, Javed; Ansys, United States

Al Ghamdi, Abdullah; Maaden Aluminium, Saudi Arabia

Al Khaldi, Ahmed; Kipic, Kuwait

\title{
TMS Welcomes New Members
}

The TMS Board of Directors approved professional membership for the following individuals at its June $\mathbf{2 0 1 8}$ meeting. Please join us in congratulating and welcoming them to all the privileges and benefits of TMS membership.
AlFadhalah, Khaled; Kuwait University, Kuwait

Allain, Sebastien; Institut Jean Lamour/Mines Nancy, France

Allaire, Claude; CIR Laboratory Inc, Canada

Allen, Kazumi; Oak-Mitsui Technologies, LLC, United States

Alshawarghi, Hamzah; Praxair, United States

Al-Taher, Ahmed; Maaden Aluminium, Saudi Arabia

Altinsel, Yigit; Meta Nikel Kobalt A.S., Turkey

Altuner, Hatice Mollaoglu; Assan Aluminum, Turkey

Alyoubi, Mohammad; Saudi Arabia

Amankwah, Isaac; Volta Aluminium Co. Ltd, Ghana

Anai, Kei; Mitsui Mining \& Smelting Co. Ltd, Japan

Anderoglu, Osman; The University of New Mexico Nuclear Engineering, United States

Andrieu, Sandra; Safran Landing Systems, France

Andritsos, Eleftherios; King's College London, United Kingdom

Angart, Samuel Gilbert; Arconic Howmet Research Center, United States

Antillon, Edwin; UES Inc, United States

Ao, Zhimin; Guangdong University of Technology, China

Aparicio, Conrado; University of Minnesota, United States
Aphale, Ashish; University of Connecticut, United States

Asad, Nader; Aluminium Bahrain, Bahrain

Ashcroft, lan; University of Nottingham, United Kingdom

Aslani, Arash; N2 Biomedical, United States

Augustyn, Boguslaw; Institute of Non Ferrous Metals, Poland

Azar, Amin S.; Sintef, Norway

Badowski, Mark; Hydro Aluminium Rolled Products, Germany

Badres, Samir; PT Indonesia Asahan Aluminium (Persero), Indonesia

Bae, Jun $\mathrm{Ho}$; Korea Institute of Materials Science, South Korea

Baker, Alexander; Lawrence Livermore National Laboratory, United States

Balachandran, Uthamalingam; Argonne National Laboratory, United States

Banerjee, Soumik; Washington State University, United States

Bank, Tracy; United States

Baranov, llia; Rusal, Russian Federation

Barber, Robert E.; Shear Form, Inc, United States

Barnes, Cris; Los Alamos National Laboratory, United States

Barranguet, Christiane; Elsevier, Netherlands

Bateman, Allyssa; Boise State University, United States
Batnasan, Altansukh; Akita University, Japan

Beake, Ben; Micro Materials Ltd, United Kingdom

Bean, Glenn E.; The Aerospace Corporation, United States

Becasse, Sebastien; Rio Tinto Aluminium, France

Beck, Sara; Pratt \& Whitney, United States

Becker, Michael; Technical University of Kaiserslautern, Germany

Beeh, Elmar; German Aerospace Center, Germany

Behera, Rakesh K.; New York University, United States

Bell, John Stuart; Canadian Nuclear Laboratories, Canada

Benson, Lyndsey; Metalysis, United Kingdom

Benson, Michael T.; Idaho National Laboratory, United States

Beretta, Stefano; Politecnico di Milano Mechanical Enginer, Italy

Bernier, Joel V.; Lawrence Livermore National Laboratory, United States

Bhattacharya, Somesh; National Institute for Materials Science, Japan

Bian, Mingzhe; National Institute for Materials Science, Japan

Birosca, Soren; Swansea University, United Kingdom

Birringer, Rainer; Saarland University, Germany 


\begin{tabular}{|c|c|c|c|}
\hline $\begin{array}{l}\text { Bischoff, Jeremy; } \\
\text { Framatome, France }\end{array}$ & $\begin{array}{l}\text { Brandl, Christian; Karlsruhe } \\
\text { Institute of Technology, } \\
\text { Germany }\end{array}$ & $\begin{array}{l}\text { Carmack, Jon; Idaho National } \\
\text { Laboratory, United States }\end{array}$ & $\begin{array}{l}\text { Chen, Di; Los Alamos } \\
\text { National Laboratory, } \\
\text { United States }\end{array}$ \\
\hline $\begin{array}{l}\text { Biven, Laura; U.S. } \\
\text { Department of Energy, }\end{array}$ & $\begin{array}{l}\text { Germany } \\
\text { Branicio, Paulo; University of }\end{array}$ & $\begin{array}{l}\text { Castro, Gerhard; Wagstaff, } \\
\text { United States }\end{array}$ & Chen, Gaoqiang; Tsinghua \\
\hline United States & Southern California, United & Castro, Ricardo; University & University, China \\
\hline $\begin{array}{l}\text { Blacker, Ted; Sandia } \\
\text { National Laboratories, }\end{array}$ & $\begin{array}{l}\text { States } \\
\text { Brennan, Matthew; Metallized }\end{array}$ & $\begin{array}{l}\text { of California Davis, United } \\
\text { States }\end{array}$ & $\begin{array}{l}\text { Chen, Li-Chyong; National } \\
\text { Taiwan University, Taiwan }\end{array}$ \\
\hline United States & Carbon Corporation, United & Cazacu, Oana; University of & Chen, Lidong; Shanghai \\
\hline $\begin{array}{l}\text { Blackstock, Gregory; } \\
\text { Arconic, United States }\end{array}$ & Brown, Ben R.; Kansas City & Celik, Emin; Toscelik Plate and & $\begin{array}{l}\text { Institute of Ceramics, } \\
\text { China }\end{array}$ \\
\hline Blomqvist, Andreas; Sandvik & $\begin{array}{l}\text { National Security Campus, } \\
\text { United States }\end{array}$ & Sheet Ind Co, Turkey & Chen, Shikui; State \\
\hline $\begin{array}{l}\text { Boczkal, Sonia; Institute } \\
\text { Bent R\&, sweden }\end{array}$ & Brown, Jeremy; Bridgnorth & $\begin{array}{l}\text { Celik, Osman Halil Osman; } \\
\text { Asas Aluminum, Turkey }\end{array}$ & $\begin{array}{l}\text { University of New York, } \\
\text { United States }\end{array}$ \\
\hline $\begin{array}{l}\text { of Non Ferrous Metals, } \\
\text { Poland }\end{array}$ & Kingdom & Celikin, Mert; University & $\begin{array}{l}\text { Chen, Silian; China Iron \& } \\
\text { Steel Research Institute, }\end{array}$ \\
\hline Bogle, Stephanie; U.S. & $\begin{array}{l}\text { Brown, Ryan; Norican Group, } \\
\text { United States }\end{array}$ & Celine, Varvenne; CNRS Aix- & China \\
\hline Agency, United States & $\begin{array}{l}\text { Budiansky, Noah; Exponent, } \\
\text { United States }\end{array}$ & Cha, Chong; Rolls-Royce & $\begin{array}{l}\text { Chen, Ilejun; Department } \\
\text { of Mineral Processing, }\end{array}$ \\
\hline $\begin{array}{l}\text { Boles, Steven T.; Hong Kong } \\
\text { Polytechnic University, } \\
\text { Hong Kong }\end{array}$ & $\begin{array}{l}\text { Bugnion, Louis; Kan-Nak Ltd, } \\
\text { Switzerland }\end{array}$ & $\begin{array}{l}\text { Corporation, United States } \\
\text { Chai, Dengpeng; Chalco }\end{array}$ & $\begin{array}{l}\text { Science \& Technology, } \\
\text { China }\end{array}$ \\
\hline $\begin{array}{l}\text { Boll, Torben; Karlsruhe } \\
\text { Institute of Technology, } \\
\text { Germany }\end{array}$ & $\begin{array}{l}\text { Bunning, Timothy; Air Force } \\
\text { Research Laboratory, } \\
\text { United States }\end{array}$ & $\begin{array}{l}\text { Zhengzhou Research } \\
\text { Institute, China } \\
\text { Chalavadi, Pradeep; Ansys Inc, }\end{array}$ & $\begin{array}{l}\text { Chen, Xing-Qiu; Institute Of } \\
\text { Metal Research, Chinese } \\
\text { Academy of Sciences, }\end{array}$ \\
\hline $\begin{array}{l}\text { Bonora, Nicola; University of } \\
\text { Cassino, Italy }\end{array}$ & $\begin{array}{l}\text { Burkhardt, Irmela; Helmholtz- } \\
\text { Zentrum Geesthacht, } \\
\text { Germany }\end{array}$ & $\begin{array}{l}\text { Chaleby, Nathen; Hydro, United } \\
\text { States }\end{array}$ & $\begin{array}{l}\text { Chmelik, Frantisek; Charles } \\
\text { University, Czech }\end{array}$ \\
\hline $\begin{array}{l}\text { Borchers, Tyler; Arconic, } \\
\text { United States }\end{array}$ & $\begin{array}{l}\text { Burn, Graham; Novelis UK Ltd, } \\
\text { United Arab Emirates }\end{array}$ & $\begin{array}{l}\text { Chan, Isaac; U.S. Department } \\
\text { of Energy, United States }\end{array}$ & Cho, Jae-Ik; Korea Institute \\
\hline $\begin{array}{l}\text { Bordeenithikase, Punnathat; } \\
\text { NASA Jet Propulsion }\end{array}$ & $\begin{array}{l}\text { Burr, Patrick; University of } \\
\text { New South Wales Sydney, }\end{array}$ & $\begin{array}{l}\text { Chang, Jiyoung; University of } \\
\text { Utah, United States }\end{array}$ & $\begin{array}{l}\text { of Industrial Technology, } \\
\text { South Korea }\end{array}$ \\
\hline $\begin{array}{l}\text { Laboratory/California } \\
\text { Institute of Technology, }\end{array}$ & Australia & Chang, Shou-Yi; National Tsing & $\begin{array}{l}\text { Choi, Shi Hoon; Sunchon } \\
\text { National University, South }\end{array}$ \\
\hline Rorkn & $\begin{array}{l}\text { Butcher, Cilitord, University of } \\
\text { Waterloo, Canada }\end{array}$ & Chang. Ying: Dalian University & \\
\hline $\begin{array}{l}\text { BorkowSkl, LuKe; United } \\
\text { Technologies Research } \\
\text { Center, United States }\end{array}$ & $\begin{array}{l}\text { Byerly, Kevin; National Energy } \\
\text { Technology Laboratory / }\end{array}$ & $\begin{array}{l}\text { of Technology, China } \\
\text { Chang. Yuan-Wei; National }\end{array}$ & $\begin{array}{l}\text { Choi, Suk-Ho; Kyung Hee } \\
\text { University, South Korea }\end{array}$ \\
\hline $\begin{array}{l}\text { Botu, Venkatesh; Corning } \\
\text { Inc, United States }\end{array}$ & $\begin{array}{l}\text { AECOM, United States } \\
\text { Cairney, Julie M.; The }\end{array}$ & $\begin{array}{l}\text { Chiao Tung University, } \\
\text { Taiwan }\end{array}$ & $\begin{array}{l}\text { Chomyn, Kyle; Hatch, } \\
\text { Canada }\end{array}$ \\
\hline $\begin{array}{l}\text { Bourgier, Arnaud; Rio Tinto } \\
\text { Aluminium, France }\end{array}$ & $\begin{array}{l}\text { University of Sydney, } \\
\text { Australia }\end{array}$ & $\begin{array}{l}\text { Chaodong, Liu; Shenyang } \\
\text { Aluminum \& Magnesium }\end{array}$ & $\begin{array}{l}\text { Chou, Kevin; University of } \\
\text { Louisville, United States }\end{array}$ \\
\hline $\begin{array}{l}\text { Bourque, Eric; Aluminerie } \\
\text { Alouette, Canada }\end{array}$ & $\begin{array}{l}\text { Calta, Nicholas; Lawrence } \\
\text { Livermore National } \\
\text { Laboratory, United States }\end{array}$ & $\begin{array}{l}\text { Engineering \& Research } \\
\text { Institute Co., Ltd, China }\end{array}$ & $\begin{array}{l}\text { Chou, Mei-Yin; Academia } \\
\text { Sinica, Taiwan }\end{array}$ \\
\hline $\begin{array}{l}\text { Boussinot, Guillaume; } \\
\text { Access E.V., Germany } \\
\text { Bowden, David; University }\end{array}$ & $\begin{array}{l}\text { Cantwell, Patrick R.; Rose- } \\
\text { Hulman Institute of } \\
\text { Technology, United States }\end{array}$ & $\begin{array}{l}\text { Charpagne, Marie-Agathe; } \\
\text { University of California } \\
\text { Santa Barbara, United } \\
\text { States }\end{array}$ & $\begin{array}{l}\text { Chowdhury, Sugata; } \\
\text { National Institute } \\
\text { of Standards and } \\
\text { Technology, United }\end{array}$ \\
\hline $\begin{array}{l}\text { of Manchester, United } \\
\text { Kingdom }\end{array}$ & $\begin{array}{l}\text { Cao, Qingping; Zhejiang } \\
\text { University, China }\end{array}$ & $\begin{array}{l}\text { Chasse, Kevin R.; Northrop } \\
\text { Grumman Corporation, }\end{array}$ & States \\
\hline $\begin{array}{l}\text { Bowman, Keith J.; University } \\
\text { of Maryland Baltimore } \\
\text { County, United States }\end{array}$ & $\begin{array}{l}\text { Capps, Nathan; Structural } \\
\text { Integrity Associates, United } \\
\text { States }\end{array}$ & $\begin{array}{l}\text { United States } \\
\text { Chaswal, Vibhor; University of } \\
\text { Cincinnati, United States }\end{array}$ & $\begin{array}{l}\text { Christ, Hans Juergen; } \\
\text { Universitaet Siegen, } \\
\text { Germany }\end{array}$ \\
\hline $\begin{array}{l}\text { Bradley, Edwin L.; Motorola, } \\
\text { United States }\end{array}$ & $\begin{array}{l}\text { Caram, Rubens; University of } \\
\text { Campinas, Brazil }\end{array}$ & $\begin{array}{l}\text { Chen, Dan; Harbin Engineering } \\
\text { University, China }\end{array}$ & $\begin{array}{l}\text { University of Technology, } \\
\text { Poland }\end{array}$ \\
\hline
\end{tabular}




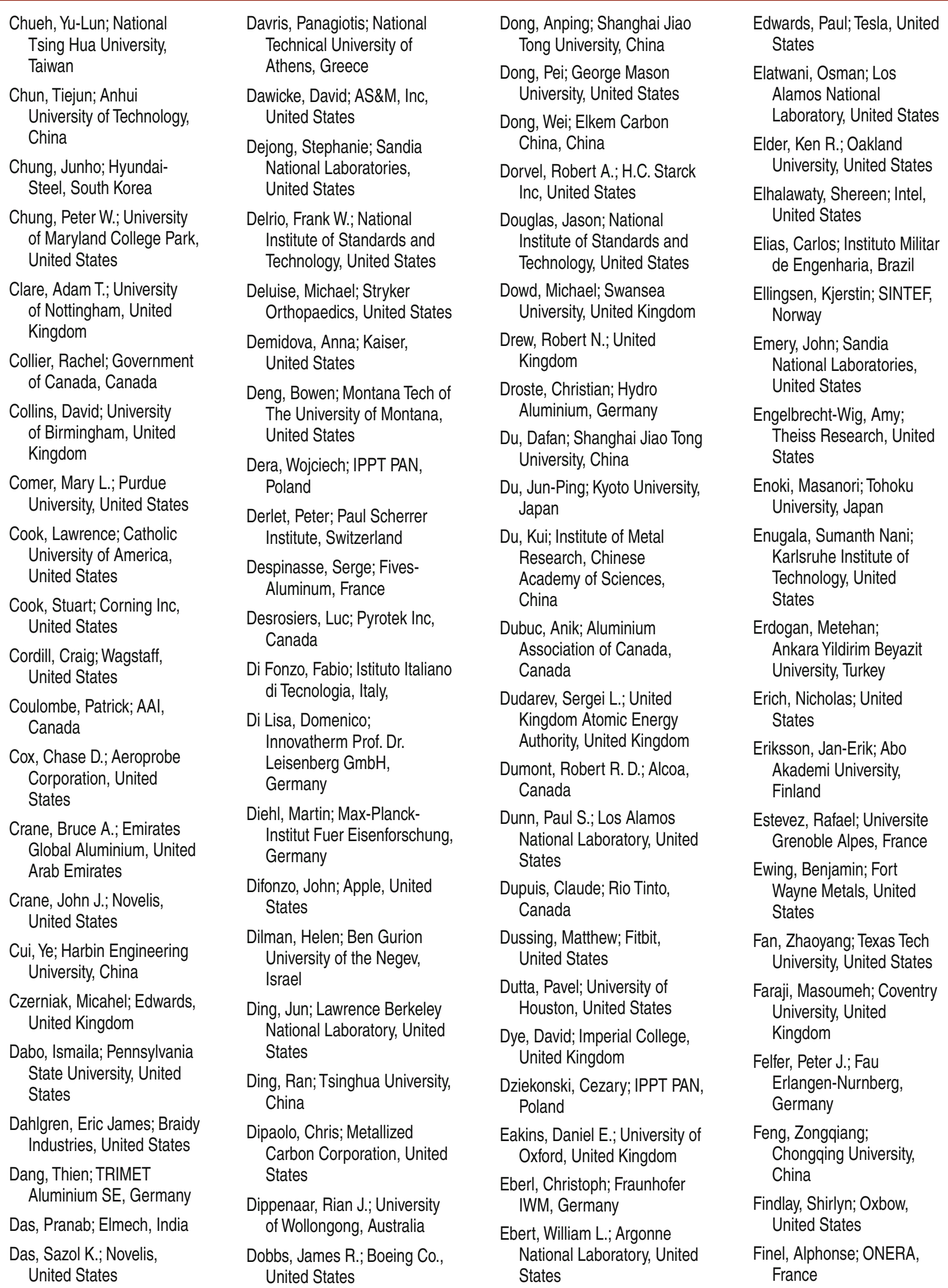




\begin{tabular}{|c|c|c|c|}
\hline $\begin{array}{c}\text { Flater, Philip J.; Air Force } \\
\text { Research Laboratory, }\end{array}$ & $\begin{array}{l}\text { Gao, Shoulei; Sunstone } \\
\text { Development, China }\end{array}$ & $\begin{array}{l}\text { Gordon, John; JG Novel } \\
\text { Solutions, United States }\end{array}$ & $\begin{array}{l}\text { Harahap, Rainaldy; PT } \\
\text { Indonesia Asahan }\end{array}$ \\
\hline United States & Gao, Wang; Jilin University, & $\begin{array}{l}\text { Grabowski, Blazej; Max- } \\
\text { Planck-Institut, Germany }\end{array}$ & $\begin{array}{l}\text { Aluminium (Persero), } \\
\text { Indonesia }\end{array}$ \\
\hline $\begin{array}{l}\text { Fioro, Jerrold A.; University } \\
\text { of Virginia, United States }\end{array}$ & $\begin{array}{l}\text { China } \\
\text { Garcia Ferre, Francisco; ABB }\end{array}$ & Graefe, Markus; Emirates & Harp, Jason; Idaho National \\
\hline $\begin{array}{l}\text { Fontes Vieira, Carlos; State } \\
\text { University of the North }\end{array}$ & Schweiz AG, Switzerland & $\begin{array}{l}\text { Global Aluminium, United } \\
\text { Arab Emirates }\end{array}$ & $\begin{array}{l}\text { Laboratory, United States } \\
\text { Harte, Allan; University }\end{array}$ \\
\hline Fluminense, Brazil & $\begin{array}{l}\text { Garner, Alistair; University } \\
\text { of Manchester, United }\end{array}$ & $\begin{array}{l}\text { Griebel, Adam; Fort Wayne } \\
\text { Metals, United States }\end{array}$ & $\begin{array}{l}\text { of Manchester, United } \\
\text { Kingdom }\end{array}$ \\
\hline $\begin{array}{l}\text { Ford, Kurtis; Sandia National } \\
\text { Laboratories, United } \\
\text { States }\end{array}$ & $\begin{array}{l}\text { Gault, Baptiste; Max- } \\
\text { Planck Institute Fuer }\end{array}$ & $\begin{array}{l}\text { Grilli, Nicolo; Purdue University, } \\
\text { United States }\end{array}$ & $\begin{array}{l}\text { Hartwig, Karl Ted; Texas } \\
\text { A\&M University, United } \\
\text { States }\end{array}$ \\
\hline $\begin{array}{l}\text { Forrest, David R.; U.S. } \\
\text { Department of Energy, } \\
\text { United States }\end{array}$ & $\begin{array}{l}\text { Eisenforschung, Germany } \\
\text { Gavini, Vikram; University of } \\
\text { Michigan, United States }\end{array}$ & $\begin{array}{l}\text { Gros, Nicolas; Carbone Savoie, } \\
\text { France } \\
\text { Gruen, Nicole; Medical }\end{array}$ & $\begin{array}{l}\text { Haskins, Adam W.; Forensic } \\
\text { Metallurgy LLC, United } \\
\text { States }\end{array}$ \\
\hline $\begin{array}{l}\text { Forrest, Delwyn; Alcoa, } \\
\text { United States }\end{array}$ & $\begin{array}{l}\text { Gaynor, Andrew; U.S. Army } \\
\text { Research Laboratory, }\end{array}$ & $\begin{array}{l}\text { University of Graz, Austria } \\
\text { Guelou, Gabin; National }\end{array}$ & $\begin{array}{l}\text { Haskins, Justin; NASA , } \\
\text { United States }\end{array}$ \\
\hline $\begin{array}{l}\text { Forzley, Alfred; Lockheed } \\
\text { Martin, United States }\end{array}$ & United States & $\begin{array}{l}\text { Institute for Materials } \\
\text { Science, Japan }\end{array}$ & $\begin{array}{l}\text { Hatem, Hamad; Aluminum } \\
\text { Bahrain, Bahrain }\end{array}$ \\
\hline $\begin{array}{l}\text { Foster, lan; University } \\
\text { of Chicago/Argonne }\end{array}$ & $\begin{array}{l}\text { Technological University, } \\
\text { United States }\end{array}$ & $\begin{array}{l}\text { Gueninchault, Nicolas; Xnovo } \\
\text { Technology, Denmark }\end{array}$ & $\begin{array}{l}\text { He, Jianhong; Oerlikon } \\
\text { Metco, United States }\end{array}$ \\
\hline $\begin{array}{l}\text { National Laboratory, } \\
\text { United States }\end{array}$ & Gerard, Celine; Institut Pprime & $\begin{array}{l}\text { Gulizia, Stefan; CSIRO } \\
\quad \text { Manufacturing, Australia }\end{array}$ & $\begin{array}{l}\text { Heamon, Mark L.; SELEE } \\
\text { Corporation. United }\end{array}$ \\
\hline $\begin{array}{l}\text { Foulatier, Elisabeth; Fontes } \\
\text { Refractories, France }\end{array}$ & Ghahreman, Ahmad; Queen's & $\begin{array}{l}\text { Guraya, Teresa; University of } \\
\text { the Basque Country, Spain }\end{array}$ & $\begin{array}{l}\text { States } \\
\text { Heckman. Nathan: Sandia }\end{array}$ \\
\hline $\begin{array}{l}\text { Fragner, Werner; AMAG } \\
\quad \text { Austria Metall AG, Austria }\end{array}$ & $\begin{array}{l}\text { Ghosh, Avik; University of } \\
\text { Virginia. United States }\end{array}$ & $\begin{array}{l}\text { Ha, Changwan; Helmholtz- } \\
\text { Zentrum Geesthacht, }\end{array}$ & $\begin{array}{l}\text { National Laboratories, } \\
\text { United States }\end{array}$ \\
\hline $\begin{array}{l}\text { Fujii, Satoshi; Tokyo Institute } \\
\text { of Technology, Japan }\end{array}$ & Ghosh, Madan Mohan; & Haga, Yasufumi; JX & $\begin{array}{l}\text { Heine, Reed; Linde, United } \\
\text { States }\end{array}$ \\
\hline $\begin{array}{l}\text { Fukumoto, Takumi; Kobe } \\
\text { University, Japan }\end{array}$ & Technology Durgapur, India & $\begin{array}{l}\text { Nippon Mining \& Metals } \\
\text { Corporation, Japan }\end{array}$ & $\begin{array}{l}\text { Hell, Jean-Christophe; } \\
\text { Arcelormittal Global R\&D }\end{array}$ \\
\hline $\begin{array}{l}\text { Furukawa, Atsushi; Honda } \\
\text { R\&D Co., Ltd, Japan }\end{array}$ & $\begin{array}{l}\text { Gilbert, Mark; United Kingdom } \\
\text { Atomic Energy Authority, } \\
\text { United Kingdom }\end{array}$ & $\begin{array}{l}\text { Hamar, Vladimir; Slovalco A.S., } \\
\text { Slovakia }\end{array}$ & $\begin{array}{l}\text { France } \\
\text { Hemmatian, Zahra; }\end{array}$ \\
\hline $\begin{array}{l}\text { Gaffar, Ahmed; Aluminum } \\
\text { Bahrain, India }\end{array}$ & Giri, Anit; U.S. Army Research & $\begin{array}{l}\text { Hamby, Eric; Corning Inc, } \\
\text { United States }\end{array}$ & $\begin{array}{l}\text { University of California, } \\
\text { United States }\end{array}$ \\
\hline $\begin{array}{l}\text { Gafner, Alyssa; U.S. Army } \\
\text { TARDEC, United States }\end{array}$ & Goho & $\begin{array}{l}\text { Hamoush, Sameer; North } \\
\text { Carolina A\&T State }\end{array}$ & $\begin{array}{l}\text { Hendrickson, Mandana; } \\
\text { TIMET, United States }\end{array}$ \\
\hline Galles, Daniel; U.S. Army & Japan & Han, Jeong; Inha University, & $\begin{array}{l}\text { Henry, Chad; GKN Additive, } \\
\text { United States }\end{array}$ \\
\hline $\begin{array}{l}\text { Research Laboratory, } \\
\text { United States }\end{array}$ & $\begin{array}{l}\text { Gokelma, Mertol; Norwegian } \\
\text { University of Science and }\end{array}$ & $\begin{array}{l}\text { South Korea } \\
\text { Han, Yihua; North China }\end{array}$ & $\begin{array}{l}\text { Heo, Yongkang; Hyundai- } \\
\text { Steel, South Korea }\end{array}$ \\
\hline $\begin{array}{l}\text { Gamble, Kyle; Idaho National } \\
\text { Laboratory, United States }\end{array}$ & $\begin{array}{c}\text { Technology, Norway } \\
\text { Golden, Patrick; Air Force }\end{array}$ & $\begin{array}{l}\text { University of Science and } \\
\text { Technology, China }\end{array}$ & $\begin{array}{l}\text { Hermann, Raphael; } \\
\text { Oak Ridge National }\end{array}$ \\
\hline $\begin{array}{l}\text { Gang, Florian; Robert Bosch } \\
\text { GmbH, Germany }\end{array}$ & $\begin{array}{l}\text { Research Laboratory, } \\
\text { United States }\end{array}$ & $\begin{array}{l}\text { Hannay, Jonathan; Naval } \\
\text { Nuclear Laboratory, United }\end{array}$ & $\begin{array}{l}\text { Laboratory, United States } \\
\text { Hernandez, Javier; }\end{array}$ \\
\hline $\begin{array}{l}\text { Gangireddy, Sindhura; } \\
\text { University of North Texas, } \\
\text { United States }\end{array}$ & $\begin{array}{l}\text { Golumbfskie, William J.; Naval } \\
\text { Surface Warfare Center } \\
\text { Carderock Division, United }\end{array}$ & $\begin{array}{l}\text { States } \\
\text { Hanson, Keenan; Stryker } \\
\text { Orthopaedics, United States }\end{array}$ & $\begin{array}{l}\text { Universidad de Sonora, } \\
\text { Mexico } \\
\text { Herper, Heike C.; Uppsala }\end{array}$ \\
\hline $\begin{array}{l}\text { Gao, Meng; University of } \\
\text { Wisconsin-Madison, } \\
\text { United States }\end{array}$ & $\begin{array}{l}\text { States } \\
\text { Gong, Pan; Huazhong } \\
\text { University of Science and }\end{array}$ & $\begin{array}{l}\text { Hanwell, Marcus; Kitware, } \\
\text { United States } \\
\text { Hao, Nanjing; Dartmouth }\end{array}$ & $\begin{array}{l}\text { University, Sweden } \\
\text { Heuser, Brent; University of } \\
\text { Illinois, United States }\end{array}$ \\
\hline $\begin{array}{l}\text { Gao, Pu-Xian; University } \\
\text { of Connecticut, United } \\
\text { States }\end{array}$ & $\begin{array}{l}\text { Technology, China } \\
\text { Gong, Peng; The University of } \\
\text { Sheffield, United Kingdom }\end{array}$ & $\begin{array}{l}\text { College, United States } \\
\text { Hara, Takaaki; Hitachi, Ltd, } \\
\text { Japan }\end{array}$ & $\begin{array}{l}\text { Hill, Michael; University of } \\
\text { California Davis, United } \\
\text { States }\end{array}$ \\
\hline
\end{tabular}




\begin{tabular}{|c|c|c|c|}
\hline $\begin{array}{l}\text { Hills, Spencer; Argonne } \\
\text { National Laboratory, } \\
\text { United States }\end{array}$ & $\begin{array}{l}\text { Hudson, Tony; Outotec, } \\
\text { Australia } \\
\text { Hummelt. Edward J.: Eaton }\end{array}$ & $\begin{array}{l}\text { Jacobson, Nathan S.; NASA } \\
\text { Glenn Research Center, } \\
\text { United States }\end{array}$ & $\begin{array}{l}\text { Jones, Jonathan; Swansea } \\
\text { University, United } \\
\text { Kingdom }\end{array}$ \\
\hline $\begin{array}{l}\text { Hinkle, Adam R.; Sandia } \\
\text { National Laboratories, } \\
\text { United States }\end{array}$ & $\begin{array}{l}\text { Corporation, United States } \\
\text { Hunter, Abigail; Los Alamos } \\
\text { National Laboratory, United }\end{array}$ & $\begin{array}{l}\text { Jak, Evgueni; Pyrosearch, The } \\
\text { University Of Queensland, } \\
\text { Australia }\end{array}$ & $\begin{array}{l}\text { Jonsson, Par G.; KTH Royal } \\
\text { Institute of Technology, } \\
\text { Sweden }\end{array}$ \\
\hline $\begin{array}{l}\text { Hiraki, Takehito; Tohoku } \\
\text { University, Japan }\end{array}$ & $\begin{array}{l}\text { States } \\
\text { Hunter, Stevan; ON }\end{array}$ & $\begin{array}{l}\text { Jamshidinia, Mahdi; GE } \\
\text { Additive, United States }\end{array}$ & $\begin{array}{l}\text { Joshi, Simon; Dana Canada } \\
\text { Corporation, Canada }\end{array}$ \\
\hline $\begin{array}{l}\text { Hoge, Keith; Metallized } \\
\text { Carbon Corporation, } \\
\text { United States } \\
\text { Holcomb, Hal; AMG } \\
\text { Aluminum, United States }\end{array}$ & $\begin{array}{l}\text { Semiconductor, United } \\
\text { States } \\
\text { Hussein, Mahmoud; University } \\
\text { of Colorado Boulder, United } \\
\text { States }\end{array}$ & $\begin{array}{l}\text { Janda, Daniel; W. L. Gore \& } \\
\text { Associates, Inc, United } \\
\text { States } \\
\text { Janecek, Milos; Charles } \\
\text { University, Czech Republic }\end{array}$ & $\begin{array}{l}\text { Jue, Jan-Fong; Idaho } \\
\text { National Laboratory, } \\
\text { United States } \\
\text { Kaban, Ivan; IFW Dresden, } \\
\text { Germany }\end{array}$ \\
\hline $\begin{array}{l}\text { Holcombe, Emily; Naval } \\
\text { Surface Warfare Center } \\
\text { Carderock Division, }\end{array}$ & $\begin{array}{l}\text { Hwang, Jinwoo; The Ohio State } \\
\text { University, United States } \\
\text { lacobescu, Remus Ion; KU }\end{array}$ & $\begin{array}{l}\text { Jared, Bradley H.; Sandia } \\
\text { National Laboratories, } \\
\text { United States }\end{array}$ & $\begin{array}{l}\text { Kaefer, Sven; Technische } \\
\text { Universiät Darmstadt, } \\
\text { Germany }\end{array}$ \\
\hline $\begin{array}{l}\text { United States } \\
\text { Holl, Norbert; Outotec, } \\
\text { Germany }\end{array}$ & $\begin{array}{l}\text { Leuven, Belgium } \\
\text { Iannitti, Gianluca; University } \\
\text { of Cassino and Southern } \\
\text { Lazio, Italy }\end{array}$ & $\begin{array}{l}\text { Jaroni, Benjamin; Trimet } \\
\text { Aluminium, Germany } \\
\text { Jarzabek, Dariusz; IPPT PAN, } \\
\text { Poland }\end{array}$ & $\begin{array}{l}\text { Kalaga, Kaushik; Argonne } \\
\text { National Laboratory, } \\
\text { United States }\end{array}$ \\
\hline $\begin{array}{l}\text { Holmstrom, Erik; Sandvik } \\
\text { Coromant R\&D, Sweden }\end{array}$ & $\begin{array}{l}\text { Ibarra, Adrian; Claudius Peters } \\
\text { (Americas) Inc, United }\end{array}$ & $\begin{array}{l}\text { Javaid, Amjad; Canmet, } \\
\text { Natural Resources Canada, }\end{array}$ & $\begin{array}{l}\text { Kan-Dapaah, Kwabena; } \\
\text { University of Ghana, } \\
\text { Ghana }\end{array}$ \\
\hline $\begin{array}{l}\text { Holstead, Alexander; } \\
\text { Air Force Research } \\
\text { Laboratory, United States }\end{array}$ & $\begin{array}{l}\text { States } \\
\text { likubo, Satoshi; Kyushu } \\
\text { Institute of Technology, }\end{array}$ & $\begin{array}{l}\text { Canada } \\
\text { Jean Louis, Achard; } \\
\text { Constellium, France }\end{array}$ & $\begin{array}{l}\text { Kang, Munjin; Korea } \\
\text { Institute of Industrial } \\
\text { Technology, South Korea }\end{array}$ \\
\hline $\begin{array}{l}\text { Hong, Daeho; Stryker, } \\
\text { United States }\end{array}$ & $\begin{array}{l}\text { Japan } \\
\text { Ikoma, Yoshifumi; Kyushu }\end{array}$ & $\begin{array}{l}\text { Jeelani, Shaik; Tuskegee } \\
\text { University, United States }\end{array}$ & $\begin{array}{l}\text { Kang, Namhyun; Pusan } \\
\text { National University, }\end{array}$ \\
\hline $\begin{array}{l}\text { Hong, Soon Hyung; Korea } \\
\text { Advanced Institute of } \\
\text { Science \& Technology, } \\
\text { South Korea }\end{array}$ & $\begin{array}{l}\text { University, Japan } \\
\text { Iloeje, Chukwunwike; Argonne } \\
\text { National Laboratory, United } \\
\text { States }\end{array}$ & $\begin{array}{l}\text { Jennings, Ryan; KCNSC/ } \\
\text { Honeywell FM\&T, United } \\
\text { States } \\
\text { Jepson, Stewart C.; Airgas, }\end{array}$ & $\begin{array}{l}\text { South Korea } \\
\text { Kang, Seong-Hoon; Korea } \\
\text { Institute of Materials } \\
\text { Science, South Korea }\end{array}$ \\
\hline $\begin{array}{l}\text { Hong, Soon-Jik; Kongju } \\
\text { National University, South } \\
\text { Korea }\end{array}$ & $\begin{array}{l}\text { Im, Jiwoo; Korea Institute of } \\
\text { Industrial Technology, South } \\
\text { Korea }\end{array}$ & $\begin{array}{l}\text { United States } \\
\text { Jia, Yun-Fei; East China } \\
\text { University of Science and }\end{array}$ & $\begin{array}{l}\text { Kangas, Pasi; Sandvik } \\
\text { Additive Manufacturing, } \\
\text { Sweden }\end{array}$ \\
\hline $\begin{array}{l}\text { Hosch, Timothy; Arconic, } \\
\text { United States } \\
\text { Howells, Andrew; Alcereco }\end{array}$ & $\begin{array}{l}\text { Iraizoz, Martin M.; Aluar, } \\
\text { Argentina } \\
\text { Isaksen, Morten; Hydro }\end{array}$ & $\begin{array}{l}\text { Technology, China } \\
\text { Jiang, Qing; Jilin University, } \\
\text { China }\end{array}$ & $\begin{array}{l}\text { Kareh, Kristina Maria; } \\
\text { Nature Communications, } \\
\text { United Kingdom }\end{array}$ \\
\hline $\begin{array}{l}\text { Inc, Canada } \\
\text { Hoyt, Jeffrey J.; McMaster } \\
\text { University, Canada }\end{array}$ & $\begin{array}{l}\text { Aluminium AS, Norway } \\
\text { Isiksacan, Cemil; Assan } \\
\text { Aluminum, Turkey }\end{array}$ & $\begin{array}{l}\text { Jiang, Wufeng; North China } \\
\text { University of Science and } \\
\text { Technology, China }\end{array}$ & $\begin{array}{l}\text { Karlsson, Breki; HRV, } \\
\text { Iceland }\end{array}$ \\
\hline $\begin{array}{l}\text { Hu, Hongfa; University of } \\
\text { Windsor, Canada }\end{array}$ & $\begin{array}{l}\text { Islam, Md Mahbubul; Purdue } \\
\text { University, United States }\end{array}$ & $\begin{array}{l}\text { Jin, Hyunwoo H.W.; } \\
\text { ExxonMobil Research \& }\end{array}$ & $\begin{array}{l}\text { Zentrum Geesthacht, } \\
\text { Germany }\end{array}$ \\
\hline $\begin{array}{l}\text { Hu, Qing-Miao; Institute of } \\
\text { Metal Research, Chinese } \\
\text { Academy of Sciences, }\end{array}$ & $\begin{array}{l}\text { Itakura, Mitsuhiro; Japan } \\
\text { Atomic Energy Agency, } \\
\text { Japan }\end{array}$ & $\begin{array}{l}\text { Engineering, United States } \\
\text { Jin, Peng; Ohio University, } \\
\text { United States }\end{array}$ & $\begin{array}{l}\text { Kato, Hajime; Toyota Centra } \\
\text { R\&D Labs., Inc, Japan }\end{array}$ \\
\hline $\begin{array}{l}\text { China } \\
\text { Huang, Haijun Jun; }\end{array}$ & $\begin{array}{l}\text { Ito, Hirofumi; Toyota Central } \\
\text { R\&D Labs., Inc, Japan }\end{array}$ & $\begin{array}{l}\text { Jochum, Tim; Microtrac, United } \\
\text { States }\end{array}$ & $\begin{array}{l}\text { Kaurmann, Helmur, AIVIAG } \\
\text { Austria Metall AG, } \\
\text { Austria }\end{array}$ \\
\hline $\begin{array}{l}\text { Shanghai Jiao Tong } \\
\text { University, China }\end{array}$ & $\begin{array}{l}\text { Iwamoto, Fumio; Sumitomo } \\
\text { Metal Mining Co., Ltd, Japan }\end{array}$ & $\begin{array}{l}\text { Joester, Derk; Northwestern } \\
\text { University, United States }\end{array}$ & $\begin{array}{l}\text { Kawalla, Claudia; } \\
\text { Technische Universität }\end{array}$ \\
\hline $\begin{array}{l}\text { Huang, Tianlin; Chongqing } \\
\text { University, China }\end{array}$ & $\begin{array}{l}\text { Izui, Hiroshi; Nihon University, } \\
\text { Japan }\end{array}$ & $\begin{array}{l}\text { Jones, Garrett; US Magnesium, } \\
\text { United States }\end{array}$ & $\begin{array}{l}\text { Bergakademie Freiberg, } \\
\text { Germany }\end{array}$ \\
\hline $\begin{array}{l}\text { Hudson, Samuel T.; USG, } \\
\text { United States }\end{array}$ & $\begin{array}{l}\text { Jackson, Martin; The University } \\
\text { of Sheffield, United Kingdom }\end{array}$ & $\begin{array}{l}\text { Jones, Geoff; SGL Carbon / } \\
\text { IPM, Australia }\end{array}$ & $\begin{array}{l}\text { Kawanishi, Ken; IHI Inc, } \\
\text { United States }\end{array}$ \\
\hline
\end{tabular}




\begin{tabular}{|c|c|c|c|}
\hline $\begin{array}{l}\text { Kawanishi, Sakiko; Tohoku } \\
\text { University, Japan } \\
\text { Kawasaki Jason K. }\end{array}$ & $\begin{array}{l}\text { Kimel, Robert Allen; } \\
\text { Pennsylvania State } \\
\text { University, United States }\end{array}$ & $\begin{array}{l}\text { Kudzal, Andelle D.; U.S. Army } \\
\text { Research Laboratory, } \\
\text { United States }\end{array}$ & $\begin{array}{l}\text { Lee, Jung Eui; } \\
\text { MetalGenTech Co., Ltd, } \\
\text { South Korea }\end{array}$ \\
\hline $\begin{array}{l}\text { University of Wisconsin } \\
\text { Madison, United States }\end{array}$ & $\begin{array}{l}\text { King, Daniel; Imperial College, } \\
\text { United Kingdom }\end{array}$ & $\begin{array}{l}\text { Kulakov, Mykola; Advanced } \\
\text { Forming Research Centre, }\end{array}$ & $\begin{array}{l}\text { Lee, Junyi; Imperial College } \\
\text { London, United Kingdom }\end{array}$ \\
\hline $\begin{array}{l}\text { Kc, Santosh; Oak Ridge } \\
\text { National Laboratory, }\end{array}$ & $\begin{array}{l}\text { Kitchin, John; Carnegie Mellon } \\
\text { University, United States }\end{array}$ & $\begin{array}{l}\text { United Kingdom } \\
\text { Kumar, Deepak; Baker Hughes, }\end{array}$ & $\begin{array}{l}\text { Lee, Seok-Woo; University } \\
\text { of Connecticut, United }\end{array}$ \\
\hline $\begin{array}{l}\text { United States } \\
\text { Keeble, Michael; Buehler, } \\
\text { United States }\end{array}$ & $\begin{array}{l}\text { Klekovkin, Alexander; North } \\
\text { American Hoganas, United } \\
\text { States }\end{array}$ & $\begin{array}{l}\text { A GE Company, United } \\
\text { States } \\
\text { Kumar, Nilesh; North Carolina }\end{array}$ & $\begin{array}{l}\text { States } \\
\text { Lee, Shiwoo; National } \\
\text { Energy Technology }\end{array}$ \\
\hline $\begin{array}{l}\text { Keiper, Winfried; Robert } \\
\text { Bosch, Germany }\end{array}$ & Knez, Mato; CIC NanoGUNE, & $\begin{array}{l}\text { State University, United } \\
\text { States }\end{array}$ & $\begin{array}{l}\text { Laboratory / AECOM, } \\
\text { United States }\end{array}$ \\
\hline $\begin{array}{l}\text { Kendrick, Marie; Toyota } \\
\text { Manufacturing, United } \\
\text { States }\end{array}$ & $\begin{array}{l}\text { Knyazeva, Marina; Technische } \\
\text { Universität Dortmund, }\end{array}$ & $\begin{array}{l}\text { Kumar, Parikshith; W L Gore } \\
\text { and Associates, United } \\
\text { States }\end{array}$ & $\begin{array}{l}\text { Lee, Soo Yeol; Chungnam } \\
\text { National University, } \\
\text { South Korea }\end{array}$ \\
\hline $\begin{array}{l}\text { Khairallah, Saad; Lawrence } \\
\text { Livermore National }\end{array}$ & Kock, Frauke; FLSmidth & $\begin{array}{l}\text { Kuo, Chin-Lung; National } \\
\text { Taiwan University, Taiwan }\end{array}$ & $\begin{array}{l}\text { Lee, Soon Gi; POSCO, } \\
\text { United States }\end{array}$ \\
\hline $\begin{array}{l}\text { Laboratory, United States } \\
\text { Kim, Cheol-Woo; Korea } \\
\text { Institute of Industrial }\end{array}$ & $\begin{array}{l}\text { Koermann, Fritz; Delft } \\
\text { University of Technology, } \\
\text { Netherlands }\end{array}$ & $\begin{array}{l}\text { Kuromura, Tetsumune; Oak } \\
\text { Mitsui Technologies, LLC, } \\
\text { United States }\end{array}$ & $\begin{array}{l}\text { Lee, Sung Ho; Agency for } \\
\text { Defense Development, } \\
\text { South Korea }\end{array}$ \\
\hline $\begin{array}{l}\text { Technology, South Korea } \\
\text { Kim, Jaehwang; Korea } \\
\text { Institute of Industrial } \\
\text { Technology, South Korea }\end{array}$ & $\begin{array}{l}\text { Kohnert, Aaron; Los Alamos } \\
\text { National Laboratory, United } \\
\text { States }\end{array}$ & $\begin{array}{l}\text { Kwak, Jay; POSCO, South } \\
\text { Korea } \\
\text { Lammatao, Joel; Aerojet } \\
\text { Rocketdyne, United States }\end{array}$ & $\begin{array}{l}\text { Lee, Taekyung; Pusan } \\
\text { National University, } \\
\text { South Korea } \\
\text { Levin, Zachary; Air Force }\end{array}$ \\
\hline $\begin{array}{l}\text { Kim, Ji Hoon; Pusan } \\
\text { National University, South } \\
\text { Korea }\end{array}$ & $\begin{array}{l}\text { Kolbe, Matthias; German } \\
\text { Aerospace Center, Germany } \\
\text { Kondos, Peter D.; Barrick Gold }\end{array}$ & $\begin{array}{l}\text { Lan, Lihong; Guangxi } \\
\text { University for Nationalities, } \\
\text { China }\end{array}$ & $\begin{array}{l}\text { Research Laboratory, } \\
\text { United States } \\
\text { Lew, Adrian; Stanford }\end{array}$ \\
\hline Kim, Jonghyun; Chongqing & inn Canada & Lane, J Matthew D.; Sandia & University, United States \\
\hline $\begin{array}{l}\text { University, China } \\
\text { Kim, Jung-Han; Korea }\end{array}$ & $\begin{array}{l}\text { Koomen, Ronald; Corning Inc, } \\
\text { United States }\end{array}$ & $\begin{array}{l}\text { Natlonal Laboratorles, } \\
\text { United States }\end{array}$ & $\begin{array}{l}\text { Li, Cai-Fu; Osaka University, } \\
\text { Japan }\end{array}$ \\
\hline $\begin{array}{l}\text { Institute of Industrial } \\
\text { Technology, South Korea }\end{array}$ & $\begin{array}{l}\text { Korbuly, Bálint; Institute for } \\
\text { Solid State Physics and }\end{array}$ & $\begin{array}{l}\text { Lang, Guanghui; Sunstone } \\
\text { Development, China }\end{array}$ & $\begin{array}{l}\text { Li, Dajian; Karlsruhe } \\
\text { Institute of Technology, }\end{array}$ \\
\hline $\begin{array}{l}\text { Kim, Kwang Ho; Pusan } \\
\text { National University, South }\end{array}$ & $\begin{array}{l}\text { Optics, Wigner Research } \\
\text { Centre for Physics, Hungary }\end{array}$ & $\begin{array}{l}\text { Langan, Tim James; CleanTeq, } \\
\text { United States }\end{array}$ & $\begin{array}{l}\text { Germany } \\
\text { Li, Delin; }\end{array}$ \\
\hline Kim, Myounggyun; Research & $\begin{array}{l}\text { Koroly, Christopher; UTC } \\
\quad \text { Aerospace Systems, United }\end{array}$ & $\begin{array}{l}\text { Laporta, Len; Capstone } \\
\text { Headwaters, United States }\end{array}$ & $\begin{array}{l}\text { CanmetMATERIALS, } \\
\text { Canada }\end{array}$ \\
\hline $\begin{array}{l}\text { Institute of Industrial } \\
\text { Science, South Korea }\end{array}$ & States & $\begin{array}{l}\text { Larson, Bennett C.; Oak Ridge } \\
\text { National Laboratory, United }\end{array}$ & $\begin{array}{l}\text { Li, Jianguo; Tsinghua } \\
\text { University, China }\end{array}$ \\
\hline $\begin{array}{l}\text { Kim, Taehyung; Hyundai- } \\
\text { Steel, South Korea } \\
\text { Kim, Taekyung; Asan, South } \\
\text { Korea }\end{array}$ & $\begin{array}{l}\text { University, United States } \\
\text { Koval, Natalia E.; Materials } \\
\text { Physics Center MPC/CFM, } \\
\text { Spain }\end{array}$ & $\begin{array}{l}\text { States } \\
\text { Larsson, Henrik; KTH Royal } \\
\text { Institute of Technology, } \\
\text { Sweden }\end{array}$ & $\begin{array}{l}\text { Li, Jianhui; Beijing Prospect } \\
\text { Metals Trading Co., Ltd, } \\
\text { China }\end{array}$ \\
\hline $\begin{array}{l}\text { Kim, Woo Kyoung; } \\
\text { Yeungnam University, }\end{array}$ & Kratky, Igor; Slovalco, Slovakia & $\begin{array}{l}\text { Lattemann, Martina; Sandvik } \\
\text { Coromant R\&D, Sweden }\end{array}$ & $\begin{array}{l}\text { University of Science \& } \\
\text { Technology, China }\end{array}$ \\
\hline $\begin{array}{l}\text { South Korea } \\
\text { Kim, Yong-Ho; Korea Institute }\end{array}$ & $\begin{array}{l}\text { Krause, Amanda R.; Lehigh } \\
\text { University, United States }\end{array}$ & $\begin{array}{l}\text { Le Bouar, Yann M.; LEM, } \\
\text { CNRS/ONERA, France }\end{array}$ & $\begin{array}{l}\text { Li, Mo; Georgia Institute } \\
\text { of Technology, United }\end{array}$ \\
\hline $\begin{array}{l}\text { of Industrial Technology, } \\
\text { South Korea }\end{array}$ & $\begin{array}{l}\text { Kroepels, Philipp; United Bulk } \\
\text { Terminals, United States }\end{array}$ & $\begin{array}{l}\text { Ledal, Rune; Hycast AS, } \\
\quad \text { Norway }\end{array}$ & $\begin{array}{l}\text { States } \\
\text { Li, Shilei; University of }\end{array}$ \\
\hline $\begin{array}{l}\text { Kim, Youngmoo; Agency for } \\
\text { Defense Development, } \\
\text { South Korea }\end{array}$ & $\begin{array}{l}\text { Kruszewski, Miroslaw J.; } \\
\text { Warsaw University of } \\
\text { Technology, Poland }\end{array}$ & $\begin{array}{l}\text { Lee, Ho Won; Korea Institute } \\
\text { of Materials Science, South } \\
\text { Korea }\end{array}$ & $\begin{array}{l}\text { Science and Technology } \\
\text { Beijing, China } \\
\text { Li, Shun; Southern }\end{array}$ \\
\hline $\begin{array}{l}\text { Kim, Yukyoung; Chonbuk } \\
\text { University, South Korea }\end{array}$ & $\begin{array}{l}\text { Kudo, Akira; California Institute } \\
\text { of Technology, United States }\end{array}$ & $\begin{array}{l}\text { Lee, Jaesoo; Shinyoung, South } \\
\text { Korea }\end{array}$ & $\begin{array}{l}\text { University of Science and } \\
\text { Technology, China }\end{array}$ \\
\hline
\end{tabular}




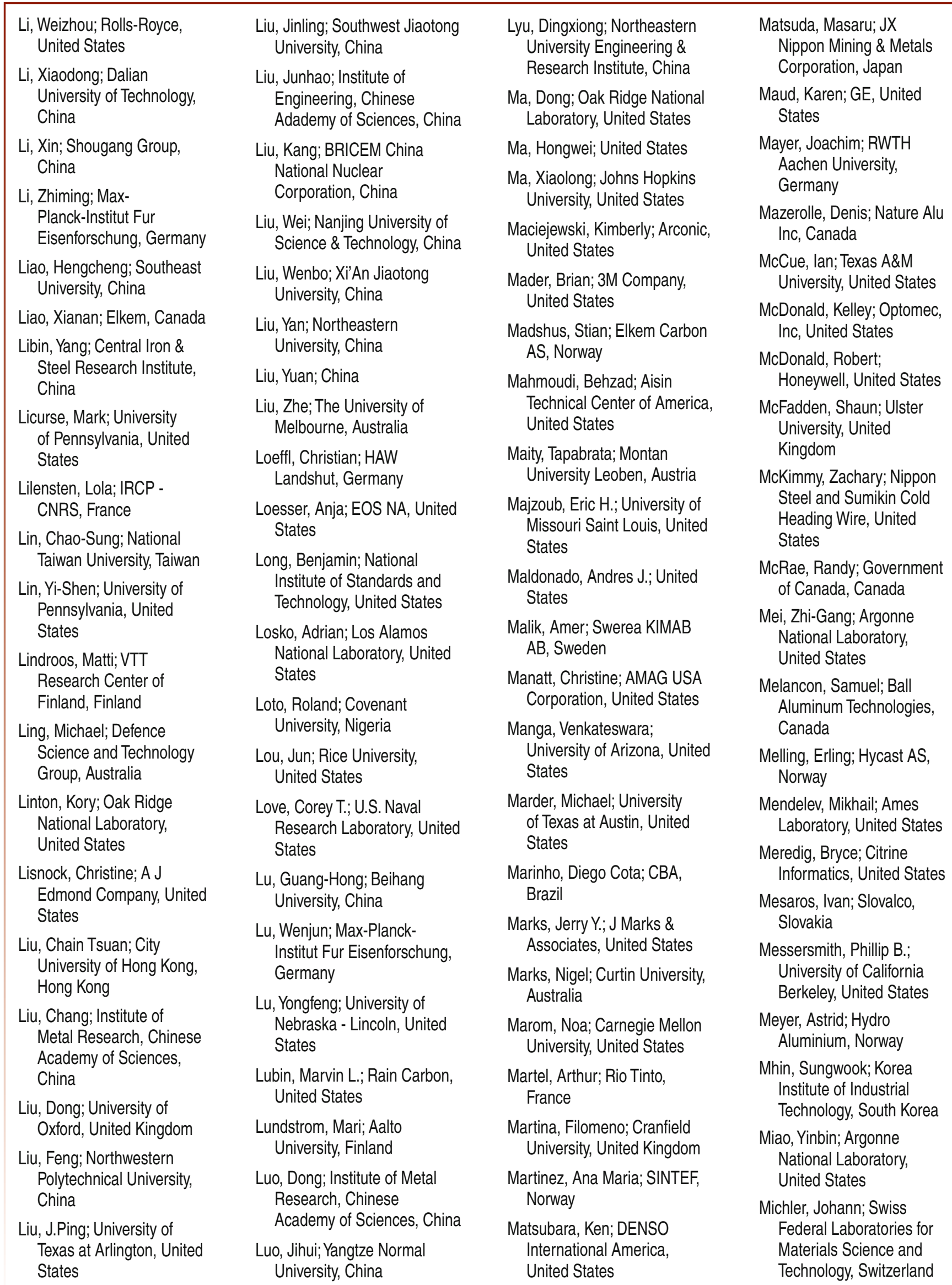




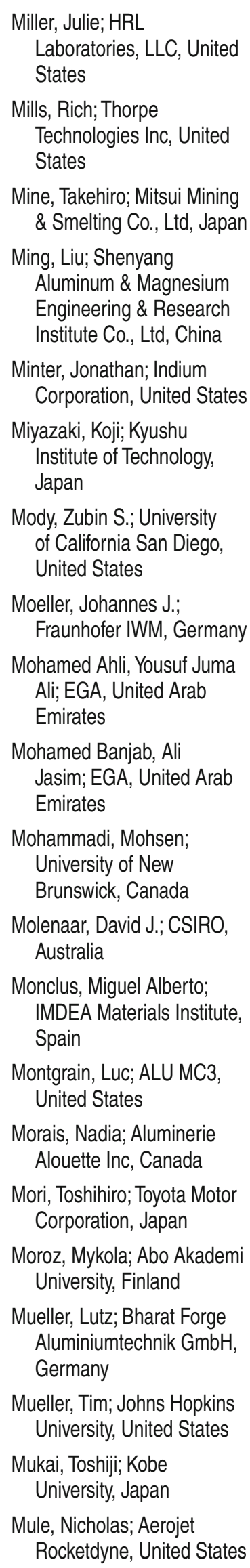

Miller, Julie; HRL Laboratories, LLC, United States

Mills, Rich; Thorpe Technologies Inc, United States

Mine, Takehiro; Mitsui Mining \& Smelting Co., Ltd, Japan

Ming, Liu; Shenyang Aluminum \& Magnesium Engineering \& Research Institute Co., Ltd, China

Minter, Jonathan; Indium Corporation, United States

Miyazaki, Koji; Kyushu Institute of Technology, Japan

Mody, Zubin S.; University of California San Diego, United States

Moeller, Johannes J.; Fraunhofer IWM, Germany

Mohamed Ahli, Yousuf Juma Ali; EGA, United Arab Emirates

Mohamed Banjab, Ali Jasim; EGA, United Arab Emirates

Mohammadi, Mohsen; University of New Brunswick, Canada

Molenaar, David J.; CSIRO, Australia

Monclus, Miguel Alberto; IMDEA Materials Institute, Spain

Montgrain, Luc; ALU MC3, United States

Morais, Nadia; Aluminerie Alouette Inc, Canada

Mori, Toshihiro; Toyota Motor Corporation, Japan

Moroz, Mykola; Abo Akademi University, Finland

Mueller, Lutz; Bharat Forge Aluminiumtechnik $\mathrm{GmbH}$, Germany

Mueller, Tim; Johns Hopkins University, United States

Mukai, Toshiji; Kobe University, Japan

Mule, Nicholas; Aerojet Rocketdyne, United States

Mullis, Andrew Martin;

University of Leeds, United Kingdom

Murakami, Yuichiro; National Institute of Advanced Industrial Science and Technology, Japan

Musinski, William D.; Air Force Research Laboratory, United States

Mutuku, Francis M.; Indium Corporation, United States

Nagata, Yoshihiko; IHI Corporation, Japan

Nagaumi, Hiromi; Soochow University, China

Nagengast, Kenneth; Coherentel Consulting, LLC, United States

Najaf Tomaraei, Golnaz; United States

Nakama, Kazuo; Sanyo Special Steel Co., Ltd, Japan

Nakamura, Mayumi; Yonekura Mfg. Co., Ltd, Japan

Nam, Alexander; Technische Universität Bergakademie Freiberg, Germany

Nam, Taehyun; Gyeongsang National University, South Korea

Naumenko, Dmitry; Forschungszentrum Juelich $\mathrm{GmbH}$, Germany

Navale, Sanket S.; Texas A\&M University, United States

Ndaba, Muzi; South32 (Mozal), Mozambique

Needleman, Alan; Texas A\&M University, United States

Nemanich, Robert J.; Arizona State University, United States

Nemir, David;TXL Group, Inc, United States

$\mathrm{Ng}$, Chee Weng; Nihon Superior Co., Ltd, Singapore

Nguyen, Quynhgiao; NASA Glenn Research Center, United States

Nian, Qiong; Arizona State University, United States
Nichols, Barbara M.; U.S. Army

Research Laboratory, United States

Nie, Zhihua; Beijing Institute of Technology, China

Ning, Jing; China

Noor, Hasan Ahmed; Aluminium Bahrain, Bahrain

Northern, Donnie L.; Novelis, United States

Novikov, Dmitri; Pratt \& Whitney, United States

Nunez, Pernelle; International Aluminium Institute, United Kingdom

Obayemi, John; Worcester Polytechnic Institute, United States

Oberdorfer, Christian; The Ohio State University, United States

O'Brien, Christopher M.; ATI Specialty Materials, United States

Odhrani, Jitendra A.; Sunpower GEN, United Arab Emirates

Oevstetun, Stale; Impec AS, Norway

Oh, Jinmok; LG Electronics, South Korea

Ohkubo, Isao; National Institute for Materials Science, Japan

Ohno, Munekazu; Hokkaido University, Japan

Oikawa, Itaru; Tohoku University, Japan

Ong, Shyue Ping; University of California San Diego, United States

Orozcocaballero, Alberto; The University of Manchester, United Kingdom

Orsund, Roar; Hydro Aluminium, Norway

Osborn, Michael; Columbus McKinnon Corporation, United States

Oskarsdottir, Gudbjorg; Iceland Aluminum Cluster, Iceland

Ott, Ryan T.; Ames Laboratory, United States
Ottinger, Deborah; U.S. Environmental Protection Agency, United States

Ouyang, Gaoyuan; lowa State University, United States

Ozturk, Tugce; Arconic, United States

Pagan, Darren; Cornell University, United States

Palanivel, Sivanesh; Tesla, United States

Panat, Rahul; Carnegie Mellon University, United States

Panchal, Jigneshkumar; A J Edmond Company, United States

Pappu, Sravani; Tesla, United States

Paquin, Michel; Rio Tinto Iron and Titanium, Canada

Park, Hojun; Hyundai-Steel, United States

Park, Hyung Wook; Ulsan National Institute of Science and Technology, South Korea

Park, Jaihyun; Research Institute of Industrial Science and Technology, South Korea

Park, Jeong-Yong; Korea Atomic Energy Research Institute, South Korea

Park, Joon; Hanbat National University, South Korea

Park, Seong Jin; Pohang University of Science and Technology, South Korea

Park, Yong Bum; Sunchon National University, South Korea

Park, Youngwoo; Portland State University, United States

Parra, Duclerc; Nuclear \& Energetic Research Institute, Brazil

Parvanian, Amir Masoud; Iran

Patel, Nishit; Dastur International Inc, Canada 
Patel, Simal; Thorpe Technologies, Inc, United States

Patrone, Paul; National Institute of Standards and Technology, United States

Paudyal, Durga; Ames Laboratory, United States

Pei, Zongrui; Oak Ridge National Laboratory, United States

Peralta, Alonso; Honeywell Aerospace, United States

Pessoa, Davi; Stihl Ferramentas Motorizadas, Brazil

Phan, Thien; National Institute of Standards and Technology, United States

Pharr, Matt; Texas A\&M University, United States

Philippi, Bastian; Dillinger, Germany

Pillai, Rishi; Forschungszentrum Juelich $\mathrm{GmbH}$, Germany

Piper, Matthew; AMG Aluminum, United Kingdom

Pistorius, Courtney; Novelis, United States

Pitera, Jed; IBM Research Almaden, United States

Plimpton, Steve J.; Sandia National Laboratories, United States

Podmaniczky, Frigyes; Wigner RCP, Hungary

Pomi, Marc A.; Estee Lauder, United States

Poplawsky, Jonathan; Oak Ridge National Laboratory, United States

Popovich, Vera; Delft University of Technology, Netherlands

Potirniche, Gabriel; University of Idaho, United States

Powell, Cynthia; Pacific Northwest National Laboratory, United States
Pramanik, Brahmananda;

Montana Tech of The University of Montana, United States

Prescenzi, Anthony D.; Ai, United States

Priddy, Matthew W.; Mississippi State University, United States

Prud'Homme, David; AluQuebec, Canada

Pusztai, Tamas; Wigner Research Centre For Physics, Hungary

Putnam, George; Scandium International Mining Corp., United States

Qian, Guoyu; Chinese Academy of Sciences, China

Qin, Junman; NFC, China

Qiu, Jie; University of Tennessee, Knoxville, United States

Qu, Haibo; Depuy Synthes, United States

Rack, Philip D.; University of Tennessee, United States

Radwitz, Sebastian; Aleris Rolled Products Germany, Germany

Ramirez, Pedro; Universidad Tecnologica de Tulancingo, Mexico

Rao, Satish I.; UES Inc, United States

Rauch, Edwin L.; Novelis, United States

Rausch, Bruno; Hydro Aluminium Deutschland $\mathrm{GmbH}$, Germany

Ravi-Chandar, Krishnaswamy; University of Texas, Unite States

Razumovskiy, Vsevolod I.; Materials Center Leoben Forschung GmbH, Austria

Reed, Evan J.; Stanford University, United States

Reedy, Earl; Sandia National Laboratories, United States

Regis, Gautier; ENSC Rennes, France
Reichardt, Ashley; SpaceX, United States

Reinke, Petra; University of Virginia, United States

Ren, Ling; Institute of Metal Research, Chinese Academy of Sciences, China

Ren, Michael; Outotec, Canada

Ren, Yu; North China Electric Power University, China

Renaudier, Steeve; Rio Tinto LRF, France

Retzlaff, Emily; United States Naval Academy, United States

Riauzi, Meretsa; PT Indonesia Asahan Aluminium (Persero), Indonesia

Richardson, Andrew; Linde, United States

Richter, Erik; P-D Refractories, Czech Republic

Ricketts, Nigel Jeffrie; Scandium International Mining Corp., Australia

Rieffer, Ariel M.; Naval Reactors, United States

Rivera, David; Lawrence Livermore National Laboratory, United States

Robbins, Joshua; Sandia National Laboratories, United States

Robertson, Blake; Hydro, United States

Robertson, John; Delta Air Lines, United States

Robichaud, Jonathan; United States

Rometsch, Paul; Monash University, Australia

Rouxel, Baptiste; Deakin University, Australia

Roy, Tania; University of Central Florida, United States

Rupp, Matthias; Fritz Haber Institute, Max Planck Society, Germany

Ryu, Ho Jin; Korea Advanced Institute of Science \& Technology, South Korea

Ryu, III; University of Texas at Dallas, United States
Ryu, Seunghwa; Korea Advanced Institute of Science \& Technology, South Korea

Saeed Alkaabi, Maryam Salem; Emirates Global Aluminium, United Arab Emirates

Saeki, Tomonori; Hitachi, Ltd, Japan

Sahara, Ryoji; National Institute for Materials Science, Japan

Salami, Mustafa; Aluminium Bahrain, Bahrain

Salamin, Sébastien; GAP Engineering, Switzerland

Salas Reyes, Antonio; National Autonomous University of Mexico, Mexico

Samolyuk, German; Oak Ridge National Laboratory, United States

Samuel, Ehab; National Research Council, Canada

Sanchez, Ida A.; Honeywell, United States

Sanchez, Jeanette; Sierra Aluminum Company, United States

Sandeman, Karl G.; The City University of New York, United States

Sandoval, Luis; AMA Inc, United States

Santoki, Jaykumar; Karlsruhe University of Applied Sciences, Germany

Sarma, Martins; HelmholtzZentrum, Germany

Sarsonova, Michaela; P-D Refractories, Czech Republic

Scardi, Paolo; University of Trento, Italy

Schablitzki, Thomas; IMR Tohoku University, Japan

Schaffer, Jeremy; Fort Wayne Metals, United States

Scheltens, Frank J.; The Ohio State University, United States 


\begin{tabular}{|c|c|c|c|}
\hline $\begin{array}{l}\text { Schroers, Jan; Yale University, } \\
\text { United States }\end{array}$ & $\begin{array}{c}\text { Sieradzki, Karl; Arizona State } \\
\text { University, United States }\end{array}$ & $\begin{array}{l}\text { Sohn, Sungwoo; Yale University, } \\
\text { United States }\end{array}$ & $\begin{array}{l}\text { Swaminathan, Viswanathan; } \\
\text { Stryker Orthopaedics, } \\
\text { United States }\end{array}$ \\
\hline $\begin{array}{l}\text { Schunk, Peter; Sandia } \\
\text { National Laboratories, } \\
\text { United States }\end{array}$ & $\begin{array}{l}\text { Sierros, Konstantinos; West } \\
\text { Virginia University, United } \\
\text { States }\end{array}$ & $\begin{array}{l}\text { Solberg, Egil; Alcoa Norway, } \\
\text { Norway }\end{array}$ & $\begin{array}{l}\text { United States } \\
\text { Swiler, Laura; Sandia National } \\
\text { Laboratories, United States }\end{array}$ \\
\hline $\begin{array}{l}\text { Schweiss, Tom; Reynolds } \\
\text { Consumer Products, } \\
\text { United States }\end{array}$ & $\begin{array}{l}\text { Silva, Edinaldo Pereira; } \\
\text { Petrocoque SA Industria e } \\
\text { Comercio, Brazil }\end{array}$ & $\begin{array}{l}\text { Son, Hyeon-Taek; Korea } \\
\text { Institute of Industrial } \\
\text { Technology, South Korea }\end{array}$ & $\begin{array}{l}\text { Ta, Yasutaka; JFE Steel } \\
\text { Corporation, Japan }\end{array}$ \\
\hline $\begin{array}{l}\text { Scott-Emuakpor, Onome; } \\
\text { Wright Patterson Air Force } \\
\text { Base, United States } \\
\text { Scurria, Matilde; Technische }\end{array}$ & $\begin{array}{l}\text { Simien, Daneesh; University } \\
\text { of Alabama at Birmingham, } \\
\text { United States } \\
\text { Sinagra, Brittany; PPG }\end{array}$ & $\begin{array}{l}\text { Sooby Wood, Elizabeth; } \\
\text { University of Texas at San } \\
\text { Antonio, United States }\end{array}$ & $\begin{array}{l}\text { Tech University, United } \\
\text { States } \\
\text { Tabuchi, Yuichiro; Nissan } \\
\text { Motor Co., Ltd, Japan }\end{array}$ \\
\hline $\begin{array}{l}\text { Universität Darmstadt, } \\
\text { Germany }\end{array}$ & $\begin{array}{l}\text { Industries, United States } \\
\text { Singh, Prashant; Ames }\end{array}$ & \multirow{2}{*}{$\begin{array}{l}\text { Spinelli, Jose; Federal } \\
\text { University of Sao Carlos, } \\
\text { Brazil }\end{array}$} & $\begin{array}{l}\text { Tafaghodi, Leili; University of } \\
\text { British Columbia, Canada }\end{array}$ \\
\hline $\begin{array}{l}\text { Sedha, Saurabh; Rio Tinto } \\
\text { Aluminum, United States }\end{array}$ & States & & $\begin{array}{l}\text { Taina, Fabio; Constellium } \\
\text { C-TEC, France }\end{array}$ \\
\hline $\begin{array}{l}\text { Sella, Serge; Aubert \& Duval, } \\
\text { France } \\
\text { Seol, Jae Bok; Pohang }\end{array}$ & $\begin{array}{l}\text { University, United States } \\
\text { Singh, Rajesh; Bharat Aluminium } \\
\text { Company Limited. India }\end{array}$ & $\begin{array}{l}\text { Srivastava, Kinshuk; Dillinger, } \\
\text { Germany }\end{array}$ & $\begin{array}{l}\text { Takajo, Shigehiro; Los Alamos } \\
\text { National Laboratory, United } \\
\text { States }\end{array}$ \\
\hline $\begin{array}{l}\text { University of Science and } \\
\text { Technology, South Korea }\end{array}$ & $\begin{array}{l}\text { Singh, Ritesh; RS + CO. LTD., } \\
\text { Canada }\end{array}$ & $\begin{array}{l}\text { States, Scott T.; Rusal America } \\
\text { Corn United States }\end{array}$ & $\begin{array}{l}\text { Tanaka, Yuta; IHI Corporation, } \\
\text { Japan }\end{array}$ \\
\hline $\begin{array}{l}\text { Sergerie, Danny; Aluminerie } \\
\text { Alouette, Canada }\end{array}$ & Sinha, Abhijit; M. N. Dastur \& & $\begin{array}{l}\text { Stewart, James; Sandia } \\
\text { National Laboratories, } \\
\text { United States }\end{array}$ & $\begin{array}{l}\text { Tang, Jie; National Insitute for } \\
\text { Material Science, Japan }\end{array}$ \\
\hline $\begin{array}{l}\text { Setyawan, Wahyu; Pacific } \\
\text { Northwest National } \\
\text { Laboratory, United States } \\
\text { Sha. Gana: Naniing }\end{array}$ & $\begin{array}{l}\text { Sjoden, Olof; ABB, Sweden } \\
\text { Skorvanek, Ivan; Institute of } \\
\text { Experimental Physics SAS, }\end{array}$ & $\begin{array}{l}\text { Stone, Morley; Air Force } \\
\text { Research Laboratory, United } \\
\text { States }\end{array}$ & $\begin{array}{l}\text { Tang, Yinglu; Swiss Federal } \\
\text { Laboratories for Materials } \\
\text { Science and Technology, } \\
\text { Switzerland }\end{array}$ \\
\hline $\begin{array}{l}\text { University of Science and } \\
\text { Technology, China }\end{array}$ & Skrotzki, Werner; Technische & \multirow{2}{*}{$\begin{array}{l}\text { Strasberg, Matthew; United } \\
\text { States } \\
\text { Strasky, Josef; Charles } \\
\text { University, Czech Republic }\end{array}$} & $\begin{array}{l}\text { Tang, Zhi; Arconic, United } \\
\text { States }\end{array}$ \\
\hline $\begin{array}{l}\text { Shah, Kinnari; LaGuardia } \\
\text { Community College - } \\
\text { CUNY, United States }\end{array}$ & $\begin{array}{l}\text { Universität Dresden, Germany } \\
\text { Sluiter, Marcel HF; Delft } \\
\text { University of Technology, }\end{array}$ & & $\begin{array}{l}\text { Taskaev, Sergey; Chelyabinsk } \\
\text { State University, Russian } \\
\text { Federation }\end{array}$ \\
\hline $\begin{array}{l}\text { Shang, Shunli; Pennsylvania } \\
\text { State University, United } \\
\text { States }\end{array}$ & $\begin{array}{l}\text { Netherlands } \\
\text { Smid, Miroslav; Paul Scherrer } \\
\text { Institut, Swaziland }\end{array}$ & $\begin{array}{l}\text { Straub, Thomas B.; Fraunhofer } \\
\text { IWM, Germany } \\
\text { Stucki, Jason: Tesla. United }\end{array}$ & $\begin{array}{l}\text { Taylor, Mary Patricia; } \\
\text { University of Birmingham, } \\
\text { United Kingdom }\end{array}$ \\
\hline $\begin{array}{l}\text { Shareef, Khalid Ahmed; } \\
\text { Aluminium Bahrain, } \\
\text { Bahrain }\end{array}$ & $\begin{array}{l}\text { Smith, Kyle; University of Illinois, } \\
\text { United States }\end{array}$ & $\begin{array}{l}\text { States } \\
\text { Stukowski, Alexander; } \\
\text { Technische Universität } \\
\text { Darmstadt, Germany }\end{array}$ & $\begin{array}{l}\text { Terrani, Kurt A.; Oak Ridge } \\
\text { National Laboratory, United } \\
\text { States }\end{array}$ \\
\hline $\begin{array}{l}\text { Sharma, Ashutosh; Ajou } \\
\text { University, South Korea }\end{array}$ & $\begin{array}{l}\text { Smith, Robert; Sierra } \\
\text { Co, United States }\end{array}$ & \multirow{3}{*}{$\begin{array}{l}\text { Su, Jie; China } \\
\text { Suave, Lorena; Safran, France } \\
\text { Suharmon, Recky; PT } \\
\quad \text { Indonesia Asahan Aluminium } \\
\text { (Persero), Indonesia }\end{array}$} & $\begin{array}{l}\text { Thenepalli, Thriveni; Hanil } \\
\text { Cement Co, Ltd, South } \\
\text { Korea }\end{array}$ \\
\hline $\begin{array}{l}\text { Shassere, Benjamin; Oak } \\
\text { Ridge National Laboratory, } \\
\text { United States }\end{array}$ & $\begin{array}{l}\text { Research Center, United } \\
\text { States } \\
\text { Smullin, Sylvia; United States }\end{array}$ & & $\begin{array}{l}\text { Thibeault, Pascal; Rio Tinto, } \\
\text { Canada }\end{array}$ \\
\hline $\begin{array}{l}\text { Shibata, Hiroyuki; IMRAM, } \\
\text { Tohoku University, Japan }\end{array}$ & $\begin{array}{l}\text { Snider, Gregory L.; University of } \\
\text { Notre Dame, United States }\end{array}$ & & $\begin{array}{l}\text { Thielman, Brett; Wagstaff, Inc, } \\
\text { United States }\end{array}$ \\
\hline $\begin{array}{l}\text { Shibuta, Yasushi; The } \\
\text { University of Tokyo, Japan }\end{array}$ & $\begin{array}{l}\text { Snyder, Jeff; Northwestern } \\
\text { University, United States }\end{array}$ & $\begin{array}{l}\text { Sun, Fusheng; Arconic, United } \\
\text { States } \\
\text { Sun, Jie; Hwapeng Precision } \\
\text { Machinery Co. Ltd, China }\end{array}$ & $\begin{array}{l}\text { Thomas, Ben; The University } \\
\text { of Sheffield, United } \\
\text { Kingdom }\end{array}$ \\
\hline $\begin{array}{l}\text { Shiotsuki, Katsuhiko; Honda } \\
\text { R\&D Co., Ltd, Japan } \\
\text { Shirayama, Sakae; The }\end{array}$ & $\begin{array}{l}\text { So, Kangpyo; Massachusetts } \\
\text { Institute of Technology, United } \\
\text { States }\end{array}$ & $\begin{array}{l}\text { Sun, Tao; Argonne National } \\
\text { Laboratory, United }\end{array}$ & $\begin{array}{l}\text { Thompson, Aidan P.; Sandia } \\
\text { National Laboratories, } \\
\text { United States }\end{array}$ \\
\hline Universily or tokyo, sapan & Sobotka, James; Southwest & \multirow{2}{*}{$\begin{array}{l}\text { Sundberg, Glenn J.; University } \\
\text { of Massachusetts Lowell, } \\
\text { United States }\end{array}$} & Thompson, John; Scandium \\
\hline $\begin{array}{l}\text { Shu, Da; Shanghai Jiao Tong } \\
\text { University, China }\end{array}$ & $\begin{array}{l}\text { Research Institute, United } \\
\text { States }\end{array}$ & & $\begin{array}{l}\text { International Mining Corp., } \\
\text { Australia }\end{array}$ \\
\hline
\end{tabular}


Todt, Andreas; Chemnitz
University of Technology,
Germany

Tong, Vivian; Imperial College London, United Kingdom

Tong, Yang; Oak Ridge National Laboratory, United States

Toppler, lan J.; U.S. Army TARDEC, United States

Trembacki, Bradley; Sandia National Laboratories, United States

Trivedi, Rahul A.; GlobalFoundries, United States

Tsai, Che-Wei; National Tsing Hua University, Taiwan

Tsakiropoulos, Panagiotis; The University of Sheffield, United Kingdom

Tsuchida, Noriyuki; University of Hyogo, Japan

Tsuda, Takuya; SEC Carbon, Japan

Tsukada, Yuhki; Nagoya University, Japan

Tucker, Jessica; The Aerospace Corporation, United States

Tuncer, Nihan; Desktop Metal, United States

Turnage, Jing; NanoMEGAS USA, United States

Ulfig, Robert; CAMECA Instruments Inc, United States

Ulysse, Patrick; Arconic, United States

Unemoto, Atsushi; Hitachi, Ltd, Japan

Uozumi, Hisao; Honda R\&D Co., Ltd, Japan

Valdevit, Lorenzo; University of California Irvine, United States

Valenzuela, Francisco

Rolando; Escola

Politecnica da

Universidade de Sao

Paulo, Brazil
Van Brutzel, Laurent; CEA Saclay, France

Van Der Zwaag, Sybrand; Delft University of Technology, Netherlands

Van Duysen, Jean Claude; EDF, France

Varghese, Oomman; University of Houston, United States

Varshney, Mukul; Bharat Aluminium Company Limited, India

Vaughan, Alan; ExxonMobil, United States

Vedani, Maurizio; Politecnico di Milano Mechanical Engineering, Italy

Velisa, Gihan; Oak Ridge National Laboratory, United States

Veluvali, Pavan L.; Institute of Applied MaterialsComputational Material Science, Germany

Venable, Jimmy; Columbus McKinnon Corp., United States

Veron, Muriel; Grenoble INP, France

Vesely, Milan; Slovalco, Slovakia

Vialle, Greg D.; SEAKR Engineering, United States

Viardin, Alexandre; Access E.V., Germany

Vinciquerra, Joseph; GE Global Research, United States

Viswanathan, Gopal; The Ohio State University, United States

Voermann, Nils; Hatch, Canada

Vogel, Eric; Georgia Institute of Technology, United States

Von Czarnowski, Peter; LMS, United States

Vorozhtsov, Alexander; Tomsk State University, Russian Federation

Vurpillot, Francois; GPM Universite Rouen, France

Wakeda, Masato; National Institute for Materials Science, Japan
Walker, Kevin; Defence Science Technology Group, Australia

Waller, David; Yara International ASA, Norway

Wang, Bingfeng; Central South University, China

Wang, Cai-Zhuang; lowa State University, United States

Wang, Fulin; University of California Santa Barbara, United States

Wang, Guannan; Texas Tech University, United States

Wang, Jian-Ping; University of Minnesota, Twin Cities, United States

Wang, Jingsong; University of Science and Technology Beijing, China

Wang, Kun; Northeastern University, China

Wang, Ping; Anhui University of Technology, China

Wang, Wanlin; Central South University, China

Wang, William Yi; Northwestern Polytechnical University, China

Wang, Xiaodong; Zhejiang University, China

Wang, Yi; Pennsylvania State University, United States

Wang, Yi; Hwapeng Precision Machinery Co. Ltd, China

Wang, Yi-Wun; National Taiwan University, Taiwan

Wang, Zhaowen; Northeastern University, China

Wang, Zhi; Institution of Process Engineering, Chinese Academy of Sciences, China

Warner, Timothy; Constellium C-TEC, France

Wasmer, Kilian; Swiss Federal Laboratories for Materials Science and Technology, Switzerland

Waters, Cindy; North Carolina A\&T State University, United States

Watts, Seth; Lawrence Livermore National Laboratory, United States
Wei, Gang; CSIRO, Australia

Wei, Zheng; Chongqing University, China

Weinberg, David; Autodesk, Inc, United States

Wen, Wei; Los Alamos National Laboratory, United States

Wen, Xingshuo; Arcelormittal Global R\&D, United States

West, Michael K.; South Dakota School of Mines \& Technology, United States

Weston, Nicholas Samuel; The University of Sheffield, United Kingdom

White, Brendan; Honda R\&D Americas, Inc, United States

Wicke, Marcel; University of Kassel, Germany,

Wilburn, David J.; Innovatherm USA Inc, United States

Williams, Christopher B.; Virginia PolytSTAR, China

Willumeit Romer, Regine; Helmholtz-Zentrum Geesthacht, Germany

Winther, Grethe; Technical University of Denmark, Denmark

Wolff, Martin; HelmholtzZentrum Geesthacht, Germany

Wood, Benjamin; Imperial College London, United Kingdom

Wood, Mitchell; Sandia National Laboratories, United States

Worsley, David; Swansea University, United Kingdom

Wright, James; Spacex, United States

Xia, Zhenhai; University of North Texas, United States

Xie, Huaqing; Northeastern University, China

Xie, Wenjie; University of Stuttgart, Germany 


\begin{tabular}{|c|c|c|c|}
\hline $\begin{array}{l}\text { Xu, Ming; Beverage Hill } \\
\text { International Inc, United }\end{array}$ & $\begin{array}{l}\text { Yeager, John D.; Los Alamos } \\
\text { National Laboratory, United }\end{array}$ & Zeng, Qiaoshi; HP & $\begin{array}{l}\text { Zheng, Jiang; Chongqing } \\
\text { University China }\end{array}$ \\
\hline & States & $\begin{array}{l}\text { Zepon, Guilherme; DEMa/ } \\
\text { UFSCar, Brazil }\end{array}$ & Zheng. Xiaoyu:Virginia \\
\hline $\begin{array}{l}\text { Xu, Peng; Westinghouse } \\
\text { Electric Company, United } \\
\text { States }\end{array}$ & $\begin{array}{l}\text { Yeo, Sunghwan; Korea Atomic } \\
\text { Energy Research Institute, } \\
\text { South Korea }\end{array}$ & $\begin{array}{l}\text { Zevalkink, Alexandra; Michigan } \\
\text { State University, United }\end{array}$ & $\begin{array}{l}\text { Polytechnic Institute, } \\
\text { United States }\end{array}$ \\
\hline $\begin{array}{l}\text { Xu, Shuozhi; University of } \\
\text { California Santa Barbara, } \\
\text { United States }\end{array}$ & $\begin{array}{l}\text { Yeole, Shivraj Narayan; VNR } \\
\text { Vignana Jyothi Institute of } \\
\text { Engineering \& Technology, }\end{array}$ & $\begin{array}{l}\text { States } \\
\text { Zhang, Dongxian; Zhejiang } \\
\text { University, China }\end{array}$ & $\begin{array}{l}\text { Zheng, Yanzhen; Hwapeng } \\
\text { Precision Machinery Co. } \\
\text { Ltd, China }\end{array}$ \\
\hline Xu, Wenchen; Harbin Institute & & Zhang, Fan; Tohoku University, & $\begin{array}{l}\text { Zhi-Quan, Liu; Institute of } \\
\text { Metal Research, Chinese }\end{array}$ \\
\hline Yamamoto, Takuya; University & Ce Co., Ltd, China & Zhang, Hao; The Institute of & $\begin{array}{l}\text { Academy of Sciences, } \\
\text { China }\end{array}$ \\
\hline $\begin{array}{l}\text { of California Santa } \\
\text { Barbara, United States }\end{array}$ & $\begin{array}{l}\text { Yoon, Kyung Joong; Korea } \\
\text { Institute of Science and }\end{array}$ & $\begin{array}{l}\text { Scientific and Industrial } \\
\text { Research, Japan }\end{array}$ & Zhong, Yunbo; Shanghai \\
\hline $\begin{array}{l}\text { Yamamura, Yuji; Sumitomo } \\
\text { Corporation, Japan }\end{array}$ & Yoon, Usok; Pohang University & $\begin{array}{l}\text { Zhang, Jian; Xi'An Jiaotong } \\
\text { University, China }\end{array}$ & Zhou, Chengshang; Central \\
\hline $\begin{array}{l}\text { Yan, Cheng; Queensland } \\
\text { University of Technology, } \\
\text { Australia }\end{array}$ & $\begin{array}{l}\text { of Science and Technology, } \\
\text { South Korea } \\
\text { Yoshida, Katsuhito; Sumitomo } \\
\text { Electric Industries, Japan }\end{array}$ & $\begin{array}{l}\text { Zhang, Jian; NFC, China } \\
\text { Zhang, Junging; University of } \\
\quad \text { Alaska Fairbanks, United }\end{array}$ & $\begin{array}{l}\text { Zhou, Ning; Cartech, United } \\
\text { States }\end{array}$ \\
\hline $\begin{array}{l}\text { Yan, Feng; University Of } \\
\text { Alabama, United States }\end{array}$ & $\begin{array}{l}\text { Yoshikawa, Takeshi; The } \\
\text { University of Tokyo, Japan }\end{array}$ & $\begin{array}{l}\text { States } \\
\text { Zhang, Kai; Monash University, }\end{array}$ & $\begin{array}{l}\text { Zhou, Qixin; The University } \\
\text { of Akron, United States }\end{array}$ \\
\hline $\begin{array}{l}\text { Yan, Na; Northwestern } \\
\text { Polytechnical University/ } \\
\text { University of California San } \\
\text { Diego, China }\end{array}$ & $\begin{array}{l}\text { You, Sihang; Helmholtz- } \\
\text { Zentrum Geesthacht, } \\
\text { Germany }\end{array}$ & $\begin{array}{l}\text { Australia } \\
\text { Zhang, Shenjia; General Cable, } \\
\text { United States }\end{array}$ & $\begin{array}{l}\text { Zhou, Shihuai; Ames } \\
\text { Laboratory, United States } \\
\text { Zhou, Wenchao; University }\end{array}$ \\
\hline $\begin{array}{l}\text { Yan, Xinyan; Alcoa, United } \\
\text { States }\end{array}$ & $\begin{array}{l}\text { Yu, Jian; U.S. Army Research } \\
\text { Laboratory, United States }\end{array}$ & $\begin{array}{l}\text { Zhang, Shuchao; Chalco } \\
\text { Zhengzhou Research }\end{array}$ & $\begin{array}{l}\text { of Arkansas, United } \\
\text { States }\end{array}$ \\
\hline $\begin{array}{l}\text { Yang, Guangyu; Northwestern } \\
\text { Polytechnical University, } \\
\text { China }\end{array}$ & $\begin{array}{l}\text { Yu, Qin; Lawrence Berkeley } \\
\text { National Laboratory, United } \\
\text { States }\end{array}$ & $\begin{array}{l}\text { Zhang, Xuan; Argonne National } \\
\text { Laboratory, United States }\end{array}$ & $\begin{array}{l}\text { Zhou, Xiaowang; Sandia } \\
\text { National Laboratories, } \\
\text { United States }\end{array}$ \\
\hline $\begin{array}{l}\text { Yang, Jiong; Shanghai } \\
\text { University, China }\end{array}$ & $\begin{array}{l}\text { Yu, Wenzhou; Chongqing } \\
\text { University, China } \\
\text { Yu, Zhengwei; Anhui University }\end{array}$ & $\begin{array}{l}\text { Zhang, Yang; Harbin } \\
\text { Engineering University, } \\
\text { China }\end{array}$ & $\begin{array}{l}\text { Zhu, Qiang; Southern } \\
\text { University of Science and } \\
\text { Technology, China }\end{array}$ \\
\hline $\begin{array}{l}\text { Yang, Y. F.; Institute of Process } \\
\text { Engineering, Chinese } \\
\text { Academy of Sciences, } \\
\text { China }\end{array}$ & $\begin{array}{l}\text { Of lechnology, China } \\
\text { Yu, Zhenzhen; Colorado School } \\
\text { of Mines, United States }\end{array}$ & $\begin{array}{l}\text { Zhang, Yongsheng; Institute } \\
\text { of Solid State Physics, } \\
\text { Chinese Academy of }\end{array}$ & $\begin{array}{l}\text { Zhu, Ting; Georgia Institute } \\
\text { of Technology, United } \\
\text { States }\end{array}$ \\
\hline $\begin{array}{l}\text { Yang, Yang; East China } \\
\text { Normal University, China }\end{array}$ & $\begin{array}{l}\text { Yuan, Yuan; Chongqing } \\
\text { University, China }\end{array}$ & Sciences, China & $\begin{array}{l}\text { Zhu, Yuanyuan; Pacific } \\
\text { Northwest National }\end{array}$ \\
\hline $\begin{array}{l}\text { Yang, Yong; China Iron \& Steel } \\
\text { Research Institute, China }\end{array}$ & $\begin{array}{l}\text { Yudho, Ivan Eko; PT Indonesia } \\
\text { Asahan Aluminium }\end{array}$ & Zhang, Zuotai; Southern & $\begin{array}{l}\text { Laboratory, United States } \\
\text { Zhukov, Arcady: Department }\end{array}$ \\
\hline $\begin{array}{l}\text { Yang, Youjian; Northeastern } \\
\text { University, China }\end{array}$ & $\begin{array}{l}\text { (Persero), Indonesia } \\
\text { Zapke, Martin; Outotec GmbH, } \\
\text { Germany }\end{array}$ & $\begin{array}{l}\text { University of Science and } \\
\text { Technology, China } \\
\text { Zhao, Fengqi: BRICEM }\end{array}$ & $\begin{array}{l}\text { of Materials Physics, } \\
\text { University of the Basque } \\
\text { Country, Spain }\end{array}$ \\
\hline $\begin{array}{l}\text { China National Nuclear } \\
\text { Corporation, China }\end{array}$ & $\begin{array}{l}\text { Zapolsky, Helena; GPM, UMR } \\
\text { 6634, France }\end{array}$ & $\begin{array}{l}\text { China National Nuclear } \\
\text { Corporation, China }\end{array}$ & $\begin{array}{l}\text { Ziegmann, Gerhard; } \\
\text { Clausthal University of }\end{array}$ \\
\hline $\begin{array}{l}\text { Yang, Zhiqing; Institute of } \\
\text { Metal Research, Chinese }\end{array}$ & $\begin{array}{l}\text { Zazueta, Miguel; Retech } \\
\text { Systems LLC, United States }\end{array}$ & $\begin{array}{l}\text { Zhao, Lei; Baker Hughes, A GE } \\
\text { Company, United States }\end{array}$ & $\begin{array}{l}\text { Technology, Germany } \\
\text { Zollfrank, Cordt:Technical }\end{array}$ \\
\hline $\begin{array}{l}\text { Academy of Sciences, } \\
\text { China }\end{array}$ & $\begin{array}{l}\text { Zebarjadi, Mona; University of } \\
\text { Virginia, United States }\end{array}$ & $\begin{array}{l}\text { Zheludkevich, Mikhail; } \\
\text { Helmholtz Zentrum }\end{array}$ & $\begin{array}{l}\text { University of Munich, } \\
\text { Germany }\end{array}$ \\
\hline $\begin{array}{l}\text { Yapici, Guney G.; Ozyegin } \\
\text { University, Turkey }\end{array}$ & $\begin{array}{l}\text { Zeng, Jianmin; Guangxi } \\
\text { University, China }\end{array}$ & $\begin{array}{l}\text { Geesthacht, Germany } \\
\text { Zheng, Guang-Ping: Hong }\end{array}$ & $\begin{array}{l}\text { Zovko, Robert; Aluminij } \\
\text { d.d. Bosnia and }\end{array}$ \\
\hline $\begin{array}{l}\text { Yasuda, Hiroyuki; Osaka } \\
\text { University, Japan }\end{array}$ & $\begin{array}{l}\text { Zeng, Jiaqing; Central Iron and } \\
\text { Steel Resarch Institue, China }\end{array}$ & $\begin{array}{l}\text { Kong Polytechnic University, } \\
\text { Hong Kong }\end{array}$ & $\begin{array}{l}\text { Herzegovinaechnic } \\
\text { Institute, United States }\end{array}$ \\
\hline
\end{tabular}

